EUROPEAN ORGANIZATION FOR NUCLEAR RESEARCH

European Laboratory for Particle Physics

Large Hadron Collider Project

LHC Project Report 456

\title{
A GENERAL MODEL FOR THERMAL, HYDRAULIC AND ELECTRIC ANALYSIS OF SUPERCONDUCTING CABLES
}

\author{
L. Bottura ${ }^{1}$, C. Rosso ${ }^{2}$, M. Breschi ${ }^{3}$
}

\begin{abstract}
In this paper we describe a generic, multi-component and multi-channel model for the analysis of superconducting cables. The aim of the model is to treat in a general and consistent manner simultaneous thermal, electric and hydraulic transients in cables. The model is devised for most general situations, but reduces in limiting cases to most common approximations without loss of efficiency. We discuss here the governing equations, and we write them in a matrix form that is well adapted to numerical treatment. We finally demonstrate the model capability by comparison with published experimental data on current distribution in a two-strand cable.
\end{abstract}

1 CERN, Division LHC, Geneva, Switzerland

2 CryoSoft, France

3 Universita' di Bologna, Italy

Presented at CHATS-Y2K: Workshop on Computation of tHermo-hydrAulic Transients in Superconductors September 6-9, 2000, Frascati, Italy

\footnotetext{
Administrative Secretariat

LHC Division

CERN

CH - 1211 Geneva 23

Switzerland

Geneva, 22 December 2000
} 


\section{Introduction}

The transient behaviour of superconducting cables is determined by intrinsically coupled thermal, hydraulic effects. This interaction is observed in dedicated experiments as well as in operating magnets. Examples of coupling are the helium induced flow that affects stability and quench propagation in force-flow cooled cables [1], [2] and the limitation found on the quench current during ramps in multi-strand cables that is thought to be caused by premature current sharing due to large current imbalances among strands [3], [4]. Not only magnet quench, but also field quality in accelerator magnets is influenced by the current distribution in the superconducting cables through interaction with the filament magnetization [5], [6]. Finally, the balance of thermal and electrical strand contacts has been found to affect greatly the stability of a cable [7], [8].

This abridged list of examples shows that it is of paramount importance to understand and possibly control the coupling between thermal, hydraulic and electric phenomena in superconducting cables. In spite of this need, as of today the quantitative impact of the coupled phenomena involving a nonuniform current distribution is not well assessed. The reason is that the coupling of phenomena makes the analytical treatment of the transient response exceedingly difficult. This is in particular true in kA-class, low-Tc superconducting cables, where the coupling among the thermal, hydraulic and electric phenomena takes place on time scales relevant for stability and operation [9]. As a consequence, many discussions on parameters affecting the phenomena involved, e.g. interstrand resistance, lack a sound basis. Experimental results are then frequently open to widely different and sometimes discordant interpretations.

We believe that the above issues are, at least in part, related to the lack of proper simulation tools that could be used to disentangle the phenomena. In 
particular while thermal and hydraulic phenomena have been addressed in a consistent manner for several years, the attempts to deal consistently with current distribution in a general model for a superconducting cable have been few and scattered [7], [8], [10]. In [9] we have advocated that a general tool, providing consistent treatment of thermal, hydraulic and electric transients is within reach using state-of-the-art numerical solvers for partial differential equations (PDE). In this paper we describe our approach to achieving this objective.

Our approach to the problem is to divide the cable in a set of domains. We then define the differential equations that govern the evolution of the state variables for each domain. The first domain is the set of the $N$ thermal components where the temperature field is described by a set of diffusion equations. The second domain is the set of $H$ cooling channels, the hydraulic components, where flow pressure, velocity and temperature are described by mass, momentum and energy conservation balances. Finally the third domain is the set of E electric, current-carrying components where the current behaviour is described by a set of semi-continuum circuital equations. The three domains are coupled explicitly through relations among the state variables, or implicitly through material properties that depend on the value of the state variables of other domains.

To derive the system of PDE, we make the fundamental assumption that the components in the cable have a large ratio of length to cross sectional dimension, so that the equations can be written in 1-D, neglecting the transverse dimensions in the cable cross section. Taking several coupled components in parallel we finally obtain a 1-D model that is topologically equivalent to the 3-D situation in the cable.

In the paper we will present the equations forming the system of PDE, and where necessary we will detail their derivation. We will then put the system 
of equations in a form convenient for numerical treatment, and broadly describe a solution strategy. The flexibility of the model is demonstrated by an application example involving the interaction among the three coupled fields.

\section{Heat conduction model}

The $N$ thermal components of a superconducting cable can be of varied nature: superconducting strands, structural components, electrical barriers, insulators. All these materials can generate Joule heat, transport heat by conduction, and exchange heat at their mutual interfaces and at the interface with a cooling medium. Assuming that the transverse dimension of each component is small with respect to its length we can write a general 1-D heat transport equation for each component $i$ :

$$
A_{i} \rho_{i} C_{i} \frac{\partial T_{i}}{\partial t}-\frac{\partial}{\partial x}\left(A_{i} k_{i} \frac{\partial T_{i}}{\partial x}\right)=\dot{q}_{i}^{\prime}+\dot{q}_{J o u l e, i}^{\prime}+\sum_{\substack{j=1 \\ j \neq i}}^{N} \frac{\left(T_{j}-T_{i}\right)}{H_{i j}}+\sum_{h=1}^{H} p_{i h} h_{i h}\left(T_{h}-T_{i}\right)
$$

where $A_{i}$ is the cross section of the component, in principle a function of position, $\rho_{i}$ its density, $C_{i}$ the specific heat, $k_{i}$ the thermal conductivity and $T_{i}$ the temperature. We allow each component to have an internal structure, assuming that the temperature within the cross section of the component is constant. This is for instance the case of a superconducting strand composed of superconducting filaments embedded in a stabilizer matrix. For each component the total cross section is obtained as sum of the partial cross sections of the constituents, The homogenised density and thermal conductivity are obtained using an area weighting, and the homogenised specific heat using a weighting based on the mass of the constituents.

The sources in Eq. (1) are the external heating term $\dot{q}_{i}^{\prime}$, the Joule heat $\dot{q}_{J o u l e, i}^{\prime}$ if the component is carrying a current, and the heat exchanged with other thermal components or coolants modeled by the last two summations in Eq. 
(1). In the case of heat exchange among thermal components we have introduced the thermal resistance per unit length $H_{i j}$ between components $i$ and $j$ (this last at temperature $T_{j}$ ). The heat exchanged with $H$ different coolant channels depends on the wetted perimeter $p_{i h}$ and heat transfer coefficient $h_{i h}$ with the coolant flowing in channel $h$ at temperature $T_{h}$. We will discuss the details of the heat transfer coefficient when dealing with the model for the hydraulic components.

\section{Boundary conditions}

Boundary conditions for the thermal problem can be of two types: prescribed temperature or prescribed heat flux. The first case, prescribed temperature, is expressed as:

$T_{i}=T_{\text {boundary }}$

where $T_{\text {boundary }}$ is the temperature at the boundary. In the case of prescribed flux we write:

$-A_{i} k_{i} \frac{\partial T_{i}}{\partial x}=q_{\text {boundary }}$

where $q_{\text {boundary }}$ is the heating power at the boundary. Adiabatic conditions are obtained if $q_{\text {boundary }}=0$.

\section{Joule heat}

The Joule heat term depends on the current carried by the cable component $I_{i}$ and on the electric field $V_{i}$ developed along its length. In general terms we can write that:

$\dot{q}_{\text {Joule }, i}^{\prime}=I_{i} V_{i}$ 
where, for consistency with the 1-D approximation made so far, we have assumed that current and electric field have the same direction. Note that this assumption is no longer exact if the current redistributes along the length of the cable. In this case additional heat is generated in the transverse resistance. As discussed later, for the scope of this discussion we neglect this effect. In the case of a purely resistive material there is a linear relation between the electric field and current density in the material:

$V_{i}=\frac{1}{A_{i} \sigma_{i}} I_{i}$

where $\sigma_{i}$ is the average electrical conductivity of the component. In the case of a component with an internal structure and several constituent materials we define an homogeneised electrical conductivity weighted according to the area.

For a component containing a superconducting material in parallel with a stabilizing shunt the relation is more complex. The electric field in a superconducting strand or cable is obtained experimentally and is usually fitted using a power law:

$V_{i}=V_{0}\left(\frac{I_{i}}{I_{c}}\right)^{n}$

The constant $V_{0}$ is the electric field set as the criterion to define the critical current $I_{c}$. The typical range for $V_{0}$ is $10^{-4}$ to $10^{-5} \mathrm{~V} / \mathrm{m}$ (corresponding to more common units of 1 to $0.1 \mu \mathrm{V} / \mathrm{cm}$ ). The constant $n$ in Eq. (6) defines the electric field dependence on current in the proximity of the $I_{c}$ transition. Strands and cables with uniform properties are characterised by a large value of $n$, of the order of 10 and above. 
To obtain a general expression for the Joule heat dissipation in the composite component containing a superconductor we distinguish the superconducting cross section $A_{s c}$ from the other (stabilizing) materials, with a total cross section $A_{s t}$. For these last we define an equivalent conductivity $\sigma_{s t}$. The total current in the component $I_{i}$ splits in a part through the superconductor $I_{s c}$ and a part in the stabilizer $I_{s t}=I_{i}-I_{s c}$ such that the longitudinal electric field in both components is identical. The split itself depends on the non linear voltage-current relation for the superconductor, which could be different from Eq. (6) as the measurements used to establish it contain the contributions of both superconductor and stabilizer to the longitudinal voltage. In principle a relation of the type of Eq. (6) can be obtained from measurements for the superconductor only, correcting for the current sharing in the stabilizer. However it can be shown that in the range of $V_{0}$ and $n$ parameters given above the current flowing in the stabilizer is small. Therefore we can safely assume that Eq. (6) is valid for the superconductor alone, substituting the total current in the component with the current in the superconductor.

The longitudinal voltage equality in the superconductor and in the stabilizer can be therefore written as follows:

$$
\frac{I_{i}-I_{s c}}{\sigma_{s t} A_{s t}}=V_{0}\left(\frac{I_{s c}}{I_{c}}\right)^{n}
$$

which is an implicit equation for the current in the superconductor. Equation (7) can be solved by an iterative technique to obtain $I_{s c}$ and the longitudinal electric field. The total Joule heat dissipation is then given by Eq. (4).

Note finally that in accordance to the power law dependence in Eq. (6), the electric field is small below the critical current density, rising very quickly to large values above $I_{c}$. For this reason this dependence is often modelled as a 
step function, with a step from zero to infinite electric field located at $I_{c}$. Here we prefer to retain the non-linear expression above for generality, still with the possibility to specialize it to the simpler case of a step in the electric field that can be obtained choosing a very large $n$ (ideally infinite).

\section{Thermal resistances}

In Eq. (1) we have introduced the thermal resistance among two thermal components $H_{i j}$ to model thermal coupling within a cable. The corresponding values can be estimated in the case of soldered cables, where the thermal coupling takes place through thermal conduction. Such an estimate is not possible in the case when the thermal coupling takes place through contact surfaces, such as in multi-strand Rutherford or bundled cables. Lacking experimental measurements of thermal resistances, estimates can be obtained assuming that the electrical and thermal contact resistances are correlated through the Wiedeman-Franz-Lorenz law [8]:

$$
H_{i j}=\frac{R_{i j}}{L_{0} T}
$$

where $R_{i j}$ is the interstrand resistance per unit length, $L_{0}$ is the Lorenz number $\left(2.4510^{-8}\left[\Omega W / \mathrm{K}^{2}\right]\right)$ and $T$ is the average temperature of the two components. In this manner we profit from the fact that the electrical resistance is a key parameter for AC loss considerations, and is therefore often available through measurements or estimates for multi-strand cables. We stress that the above approximation is justified only to evaluate orders of magnitude. The analogy to a conductive material is not necessarily verified, and important effects such as surface contact nature (e.g. sintering) or the presence of stagnant helium permeating a cable are not taken into account.

\section{Flow model}

The flow model is written for a set of $H$ parallel, 1-D channels that can exchange mass, momentum and energy among them. The coupling of the 
channels can happen either through convection heat transfer at the mutual interface, or through direct mass transfer from one flow to the other. In Appendix A we detail how to obtain the set of the three following equations for the volumetric flow $V_{h}=A_{h} v_{h}$, the pressure $p_{h}$, and the temperature $T_{h}$ of the coolant:

$$
\begin{aligned}
& \rho_{h} \frac{\partial V_{h}}{\partial t}+\frac{\rho_{h} V_{h}}{A_{h}} \frac{\partial V_{h}}{\partial x}+A_{h} \frac{\partial p_{h}}{\partial x}-\frac{\rho_{h} V_{h}^{2}}{A_{h}^{2}} \frac{\partial A_{h}}{\partial x}=-A_{h} F_{h}-\sum_{\substack{k=1 \\
k \neq h}}^{H}\left(\Gamma_{h k}^{v}-v_{h} \Gamma_{h k}^{\rho}\right) \\
& A_{h} \frac{\partial p_{h}}{\partial t}+V_{h} \frac{\partial p_{h}}{\partial x}+\rho_{h} c_{h}^{2} \frac{\partial V_{h}}{\partial x}= \\
& -\sum_{\substack{k=1 \\
k \neq h}}^{H}\left\{c_{h}^{2} \Gamma_{h k}^{\rho}+\varphi_{h}\left[\Gamma_{h k}^{e}-v_{h} \Gamma_{h k}^{v}-\left(h_{h}-\frac{v_{h}^{2}}{2}\right) \Gamma_{h k}^{\rho}\right]\right\}+\varphi_{h} V_{h} F_{h}+\varphi_{h} \dot{q}_{h}^{\prime}+\varphi_{h} \dot{q}_{c f, h}^{\prime} \\
& A_{h} \rho_{h} C_{h} \frac{\partial T_{h}}{\partial t}+\rho_{h} V_{h} C_{h} \frac{\partial T_{h}}{\partial x}+\rho_{h} \varphi_{h} C_{h} T_{h} \frac{\partial V_{h}}{\partial x}= \\
& V_{h} F_{h}-\sum_{\substack{k=1 \\
k \neq h}}^{H}\left[\Gamma_{h k}^{e}-v_{h} \Gamma_{h k}^{v}-\left(h_{h}-\frac{v_{h}^{2}}{2}-\varphi_{h} C_{h} T_{h}\right) \Gamma_{h k}^{\rho}\right]+\dot{q}_{h}^{\prime}+\dot{q}_{c f, h}^{\prime}
\end{aligned}
$$

where $A_{h}$ is the cross section of the channel (in principle variable along the length), $\rho_{h}$ is the density and $v_{h}$ is the velocity of the coolant in the channel. The equations above contain the isentropic sound speed $c_{h^{\prime}}$ the specific heat at constant volume $C_{h}$, the specific enthalpy $h_{h}$ and the Gruneisen parameter $\varphi_{h}$. Note that, as shown in Appendix A, the equations above do not contain any approximations with respect to the conservative form and they are valid for any coolant fluid.

The quantity $F_{h}$ is the friction force defined using the friction factor $f_{h}$ and the hydraulic diameter $D_{h}$ as:

$$
F_{h}=2 \rho_{h} \frac{f_{h}}{D_{h}} v_{h}\left|v_{h}\right|
$$


The quantities $\Gamma_{h k}^{\rho}, \Gamma_{h k}^{v}$ and $\Gamma_{h k}^{e}$ are the distributed sources of mass, momentum and stagnation enthalpy per unit length of channel, originating from expulsion (or injection) of helium into (or from) another channel with index $k$ and from heat exchange. Fluxes are positive if they correspond to a net massflow from channel $h$ to channel $k$. Finally the source terms $\dot{q}_{h}^{\prime}$ and $\dot{q}_{c f, h}^{\prime}$ represent respectively the heat that enters the channel $h$ per unit length through convection at the wetted perimeter and the heat flux due to the counterflow mechanism in superfluid conditions. For the moment it is convenient to maintain the sources in this general notation.

\section{Boundary conditions}

The imposition of boundary conditions to the fluid flow is a delicate matter, that should take into account the sign of the characteristics at the boundary [11]. We have found that in the non-conservative form described above it is possible to impose accurate boundary conditions in a simpler manner if we limit our choice to a closed pipe condition, or alternatively to in- and outflow into a volume at given pressure and temperature [12]. The first case (closed pipe) is imposed setting:

$V_{h}=0$

In the second case, volume in- and out-flow, we match the number of conditions imposed to the number of characteristics entering or exiting the boundary surface. In particular we have the following possibilities

Case 1. Subsonic inflow $\left(v_{h}<c_{h}\right)$.

In this case we have 2 entering characteristics, 1 exiting characteristic. Two variables are specified

$p_{h}=p_{\text {boundary }}$ 
$T_{h}=T_{\text {boundary }}$

where $p_{\text {boundary }}$ and $T_{\text {boundary }}$ are the values of pressure and temperature at the boundary.

Case 2. Supersonic inflow $\left(v_{h}>c_{h}\right)$.

For supersonic inflow we have 3 entering characteristics and no exiting characteristic. Three variables must be specified:

$p_{h}=p_{\text {boundary }}$

$T_{h}=T_{\text {boundary }}$

$V_{h}=A_{\text {boundary }} c_{\text {boundary }}$

where $c_{\text {boundary }}$ is the sound speed at the boundary and $A_{\text {boundary }}$ is the channel cross section at the boundary.

Case.3 Subsonic outflow $\left(v_{h}<c_{h}\right)$.

In this case we have 1 entering characteristic, 2 exiting characteristics, and only one variable can be specified:

$p_{h}=p_{\text {boundary }}$

Case 4. Supersonic outflow $\left(v_{h}>c_{h}\right)$.

For supersonic outflow there is no entering characteristic and 3 exiting characteristics. In this case no boundary condition can be specified. 


\section{External source terms}

The external source for the flow are represented by heat transfer at the wetted perimeter of the channel, in contact with solid walls. We write the generic source term as:

$$
\ddot{q}_{h}^{\prime}=\sum_{i=1}^{N} p_{i h} h_{i h}\left(T_{i}-T_{h}\right)
$$

where the sum is extended on the $N$ solid walls of the thermal components with index $i$ in thermal contact with the channel $h, p_{i h}$ is the wetted perimeter, $h_{i h}$ is the heat transfer coefficient and $T_{i}$ is the wall temperature.

\section{Counterflow heat exchange in superfluid helium}

The counterflow heat transport mechanism is peculiar of heat transfer in superfluid helium (or helium II). This term has a form of a non-linear diffusion [13]:

$\dot{q}_{c f, h}^{\prime}=\frac{\partial}{\partial x}\left(A_{h} \tilde{k}_{h} \frac{\partial T_{h}}{\partial x}\right)$

where $\tilde{k}_{h}$ is an equivalent thermal conductivity defined using the superfluid thermal conductivity function $\kappa_{h}$ as:

$$
\tilde{k}_{h}=\frac{\kappa_{h}}{\left(\frac{\partial T_{h}}{\partial x}\right)^{\frac{2}{3}}}
$$

\section{Transverse fluxes}

To give an explicit expression for the transverse fluxes we indicate with $v_{h k}$ the transverse velocity from channel $h$ to channel $k$, and we assume that the two channels have a boundary delimited by a perimeter $p_{h k}$ of which the fraction $\pi_{h k}$ is perforated. We have [14]: 
$\Gamma_{h k}^{\rho}=\pi_{h k} p_{h k} v_{h k} \bar{\rho}=\dot{m}_{h k}$

$\Gamma_{h k}^{v}=\pi_{h k} p_{h k} v_{h k} \bar{\rho} \bar{v}=\dot{m}_{h k} \bar{v}$

$\Gamma_{h k}^{e}=\pi_{h k} p_{h k} v_{h k} \bar{\rho}\left(\bar{h}+\frac{\bar{v}^{2}}{2}\right)+p_{h k} h_{h k}\left(T_{h}-T_{k}\right)=\dot{m}_{h k}\left(\bar{h}+\frac{\bar{v}^{2}}{2}\right)+p_{h k} h_{h k}\left(T_{h}-T_{k}\right)$

where $\dot{m}_{h k}$ is the massflow from channel $h$ to channel $k$ per unit channel length. We assume that the transverse flow between the channels can be modelled as a discharge between two volumes at different pressure [15]. The transverse flow velocity $v_{h k}$ is then given by:

$v_{h k}=\alpha_{h k}\left(p_{h}-p_{k}\right)$

where the coefficient $\alpha_{h k}$ is given by:

$\alpha_{h k}=\sqrt{\frac{2}{\bar{\rho}\left|p_{h}-p_{k}\right|}}$

The quantities $\bar{\rho}, \bar{v}$ and $\bar{h}$ in Eqs. (20) through (24) are respectively the density, flow velocity and specific enthalpy taken from the upstream conditions of the transverse flow, i.e.:

$\bar{\rho}= \begin{cases}\rho_{h} & \text { for } p_{h} \geq p_{k} \\ \rho_{k} & \text { for } p_{h}<p_{k}\end{cases}$

$\bar{v}= \begin{cases}v_{h} & \text { for } p_{h} \geq p_{k} \\ v_{k} & \text { for } p_{h}<p_{k}\end{cases}$ 
$\bar{h}= \begin{cases}h_{h} & \text { for } p_{h} \geq p_{k} \\ h_{k} & \text { for } p_{h}<p_{k}\end{cases}$

In Eq. (22) the two terms take into account the fact that energy transfer between the two channels can happen either through mass convection (first term on the r.h.s.), or through heat transfer at the boundary (second term on the r.h.s.). The heat transfer happens on the interface perimeter $p_{h k}$ with an equivalent heat transfer coefficient $h_{h k}$.

\section{Heat transfer models}

The heat transfer coefficients $h_{i h}$ between the coolant in channel $h$ and the solid wall $i$, or $h_{h k}$ between coolant flows $h$ and $k$ are computed using empirical correlations. At present this is the most general approach as it relies on experimental data. Correlation models for the heat transfer coefficient have typical data fitting accuracy in the range of some $10 \%$, and predictive capability within a factor 2 .

\section{Friction factor models}

Similarly to the heat transfer coefficient, the friction factor of the flow is computed based on empirical correlations. Correlation models for the friction factor coefficient have typical data fitting accuracy within a factor 2.

\section{Electrical model}

The electrical model adopted here focuses on a cable formed of $E$ parallel electrically conductive components characterised by a non-linear longitudinal resistance, mutual and self inductance. Within the frame of the model the generic component can be a single strands, a cable subunit, a segregated stabilizer, or any electrically conducting structural component. It is further assumed that each component has a constant current density in its cross section, and that current transfer happens along the length of the cable in a continuous manner through distributed electrical conductances. 
The equations governing the evolution of the currents in the components are derived in [9], [16] and discussed in detail in [17] in the case of a Rutherford cable. After straightforward algebra, they can be written in the following matrix form:

$\mathbf{l} \frac{\partial \mathbf{I}}{\partial t}+\mathbf{r I}-\frac{\partial}{\partial x}\left(\mathbf{c}^{-1} \frac{\partial \mathbf{I}}{\partial x}\right)=\Delta \mathbf{v}^{e x t}$

where the unknowns are the currents $I_{e}$ in the components, packed in the array I. The matrices and vectors depend on the cable geometry (e.g. inductance per unit cable length 1), its properties (e.g. transverse conductivity per unit cable length $\mathbf{c}$ ) and operating conditions (e.g. parallel resistance $\mathbf{r}$ and external voltages $\Delta \mathbf{v}^{e x t}$ per unit cable length). See [17] for more details on their structure.

\section{Boundary conditions}

The boundary conditions for the current diffusion in a cable are dominated by the details of the connection of the cable components within the coil (i.e. joints) or to the coil ends. In fact, the conditions at the boundary can be the leading effect for the current distribution along a cable. One such example is the distribution of transverse contact resistances within a joint. To avoid the significant complication that could be induced by the necessity of handling several different types of boundary conditions, we have chosen to include the details of the connections within the analysed domain. This is possible as the model described here takes into proper account longitudinal and transverse resistance variations along the cable length. For the boundary itself we have then considered only two simple limiting cases: components insulated and individually fed, or shorted together. In the first case, if the components are all insulated at the cable end, the current is imposed by an external circuit, i.e.:

$I_{e}=I_{\text {boundary }}$ 
where $I_{\text {boundary }}$ is the current at the boundary. In any case the total current must be conserved at the boundaries, and this implies that the above boundary condition can only be imposed on $E-1$ of the $E$ electrical components.

In the second case, if all components are shorted together, the voltage differences are by definition zero and the equivalent boundary condition is (see also [16] and [17] for a discussion of this boundary condition):

$$
\frac{\partial I_{e}}{\partial x}=0
$$

\section{Coupling with heat conduction model}

To model the current distribution in a cable, the electrical model needs to be coupled to the heat conduction model described earlier. This is obtained matching a set of electrical components to corresponding thermal components. The coupling between the two domains is based on the fact that the longitudinal resistance matrix $\mathbf{r}$ appearing in the electrical model depends on the temperature computed in the heat conduction model, while the current $I_{i}$ and the Joule heat generation in the thermal component depend on the current distribution computed in the electrical model. In addition if current transfer takes place between components, heat is dissipated in the transverse conductance.

The last effect is important to analyse the AC loss properties of a cable, and is included in our model using the formalism of [17]. The heating source due to current transfer is computed using the voltage differences among electric components. This calculation requires the knowledge of the space derivative of the current in each component, $\frac{\partial I_{e}}{\partial x}$, and manipulation of the transverse conductance matrix. Although conceptually simple, the calculation is rather cumbersome. For the sake of simplicity we omit here the discussion of this 
additional heat source. This is justified also considering the fact that in the case of stability and quench transients in a cable with optimised current density the heat source associated with transverse current transfer is generally small compared to the Joule heating.

Finally, and for generality, we assume in the model that the matching is not necessarily one-to-one, i.e. a single electrical component can model the current flow in several parallel thermal components, or conversely one thermal component can model the temperature evolution in several parallel electrical components. The entries in the longitudinal resistance matrix for an electrical component are computed as the parallel resistance of all the thermal components coupled to it. By analogy we distribute the current of an electrical component among the coupled thermal components according to the parallel of the longitudinal resistances.

\section{Matrix form and system solution}

The equations presented so far are numerous and cumbersome to treat singularly. It is much more convenient to write in the following compact form for a parabolic-hyperbolic system of partial differential equations amenable of unified treatment:

$\mathbf{m} \frac{\partial \mathbf{u}}{\partial t}+\mathbf{a} \frac{\partial \mathbf{u}}{\partial x}-\frac{\partial}{\partial x}\left(\mathbf{g} \frac{\partial \mathbf{u}}{\partial x}\right)+\mathbf{s u}=\mathbf{q}$

where the vector of unknowns $\mathbf{u}(x, t)$ is defined assembling the unknowns of each PDE as derived for heat conduction in the $N$ thermal componments, conservation balances in the $H$ cooling channels and current distribution among $E$ conducting materials, i.e.: 


$$
\mathbf{u}=\left[\begin{array}{c}
T_{i} \\
V_{h} \\
p_{h} \\
T_{h} \\
I_{e}
\end{array}\right]
$$

The vector $\mathbf{u}$ has therefore size $N+H+E$. The matrices appearing in Eq. (5.1) have a block structure that can be written easily identifying terms in the equations discussed in the previous sections.

For the solution of the system of PDE Eq. (31) we have chosen a finite element method in space [18] and a finite difference algorithm of the Beam-Warming family in time [19]. Practice [12] has shown that the combination of an independent space discretization and time marching algorithm provides a flexible and accurate mean to solve large systems that involve coupled strongly parabolic equations, as is the case for the thermal and electric components, and hyperbolic equations, as for hydraulic components. We have programmed the solver using Lagrangian elements with up to 6 nodes ( $5^{\text {th }}$ order interpolation). The time marching scheme has up to $4^{\text {th }}$ order accuracy, automatic step adaption and error control. High accuracy for both space and time integration is necessary to avoid growing errors such as numerical quench-back [20].

\section{An example of application}

As we hinted in the introduction, the model for the evolution of the temperature and flow has been already applied and verified in several instances against experimental data (see for instance the CHATS proceedings [21], [22]). Current distribution in superconducting cables, on the other hand, has not been extensively measured nor simulated. We have therefore concentrated on one experiment performed by Krempasky and Schmidt [23] that involves current distribution, and in particular its coupling to temperature evolution. The experiment was performed on a two-strand cable 
prepared with a $0.3 \mathrm{~mm}$ diameter, $\mathrm{NbTi} / \mathrm{Cu}$ strand. The cable was twisted with a pitch of $10 \mathrm{~mm}$ and soldered with $\mathrm{Sn}(50 \%) \mathrm{In}$. In the middle of the cable, and over a length of approximately half a twist pitch $(5 \mathrm{~mm})$, a loop with a cross section of approximately $70 \mathrm{~mm}^{2}$ was formed between the strands. The cable was wound into a test coil, with the loop placed in the coil center, normal to the coil axis. The coil was then placed in a background magnet providing an AC vertical field. The AC field caused a variation of the flux linked with the loop in the centre of the sample. This induced currents in opposite directions in the two superconducting strands, closing through the solder along the whole cable length (supercurrents). The supercurrent circulating in the centre of the sample was measured by means of a Hall plate placed in the loop. In this experiment the cable behaved as a bi-filar line with an inductance per unit length of $0.5 \mu \mathrm{H} / \mathrm{m}$. The loop in the centre of the cable length had an estimated inductance of $0.02 \mu \mathrm{H}$. The transverse conductivity per unit length was $58 \mathrm{MS} / \mathrm{m}$. Further details on the experiment, results and interpretation can be found in [23].

We have modelled the experiment with two thermal components coupled to two electrical components representing the two strands. An hydraulic component, a channel with a large cross section thermally coupled to the strands, was used to model the helium bath. Variable electrical properties (inductance and transverse conductivity) were taken along the cable length to model the presence of the extra loop in the centre of the cable. Because of symmetry, only one half of the total length was modelled.

We show in Figs. 1 through 4 the comparison of experimentally measured current and simulation results. The measurements reported in Figs. 1 and 2 were made with a sample length of $4.7 \mathrm{~m}$ and differ only for the field sweep (reported in the inset). In the case shown Fig. 3 the sample length was $1.66 \mathrm{~m}$, and the field sweep was slow enough to reach steady state conditions (see again inset in Fig. 3). In these first three cases the sample was 
superconducting throughout the transient, and the supercurrent induced could circulate freely in the sample. The agreement of measurements and simulations is excellent, especially noting that no geometrical or electrical parameters were adapted from case to case to fit data.

In the last case presented, in Fig. 4, the supercurrent was induced by a $0.65 \mathrm{~T}$ field sweep in $4 \mathrm{~s}$. Right after the end of the sweep a $4 \mathrm{~cm}$ long heater covering the center of the sample was switched on for $1.6 \mathrm{~s}$. This caused a quench of the central part of the cable, followed by a recovery as soon as the heater was switched off. The increased longitudinal resistance pushed the supercurrent out of the quenched region. The supercurrent still flowed in the unquenched length of the sample, and, as soon as the central part recovered, diffused back into the center. In this case the agreement between experimental and simulation results is still satisfactory, although for this case the simulation overestimates the peak current by $20 \%$. Examining in detail Fig. 4 we note that the maximum error is found at the end of the field sweep, i.e. before the heater is fired, and that the simulation is in good agreement with the measurement during the first second. The difference between simulation and experiment can be explained if we postulate that during the strong field sweep, and above a certain field, the strands develop a finite longitudinal resistance caused either by onset of saturation in the filaments or by AC loss (i.e. a dynamic resistance). These effects are not included explicitly in the model.

The simulations presented were run using meshes with 250 to 1500 linear or parabolic elements, and adaptive time integration with second order accuracy. The typical CPU time required to simulate the transients presented was modest, ranging from $2^{\prime}$ to $10^{\prime}$ on a DEC-Alpha processor.

\section{Conclusions}

We have presented a consistent and comprehensive model for the thermal, hydraulic and electric analysis of superconducting cables. The model, to the 
best of our knowledge, is the first that offers the possibility of a unified treatment of the coupled fields, retains a large flexibility and is suitable for analysis of long cable lengths. In spite of the significant complexity of the single equations describing the evolution of temperature, flow and current, we have finally cast them in a matrix form is simple and can be solved by a dedicated PDE solver. We have shown that the model is manageable applying it to a current distribution experiment that involves, in one case, the interaction of two of the three coupled fields (thermal and electric). In these simulations we achieved acceptable matching of the experimental data. More validation work is needed to explore the possibilities and limitations of the model, but we believe that already in its present status the range of potential applications is very large, from detailed analysis of cable transients to assistance in the design of new cable configurations, from stability margin calculation to consistent analysis of joints.

\section{Acknowledgements}

The experimental data used for benchmarking the model were kindly provided by C. Schmidt, FzK, Germany.

\section{References}

[1] J.W. Lue, J.R. Miller, L. Dresner, Stability of Cable-in-Conduit Superconductors, J. Appl. Phys., 51, 772, 1980.

[2] L. Dresner, Stability of Superconductors, Plenum Press, 1995.

[3] M.M. Steeves, et al., Test Results from the Nb3Sn US-Demonstration Poloidal Coil, Adv. Cryo. Eng., 37 A, 345-354, 1991.

[4] A. Devred, T. Ogitsu, Ramp-rate Sensitivity of SSC Dipole Magnet Prototypes, Frontiers of Accelerator Technology, World Scientific, 184308, 1996.

[5] R. Stiening, A Possible Mechanism for Enhanced Persistent Current Sextupole Decay in SSC Dipoles, SSCL-359, 1991. 
[6] R. Wolf, The Decay of the Field Integral in Superconducting Accelerator Magnets Wound with Rutherford Cables, Proc. of $15^{\text {th }}$ Int. Mag. Tech. Conf., Beijing, pp. 238-241, Science Press, 1998.

[7] N. Amemiya, H. Yonekawa, T. Ogitsu, E. Kobayashi, K. Sasaki, N. Ohuchi, K. Tsuchiya, K. Miyashita, Influence of Current Re-distribution on Minimum Quench Energy of Superconducting Triplex Cable Against Local Disturbance, Cryogenics, 38 (5), 559-568, 1998.

[8] M.N.Wilson, R.Wolf, Calculation of Minimum Quench Energies in Rutherford Cables, IEEE Trans. Appl. Sup., 7 (2), 950-953, 1997.

[9] L. Bottura, Modelling Stability in Superconducting Cables, Physica C, 310(14) 316-326, 1998.

[10] N. Mitchell, Analysis of Non-Uniform Current Distribution Effects in Multistage Cable-in-Conduit Conductors, Cryogenics 39, 539-556, 1999.

[11] C. Hirsch, Numerical Computation of Internal and External Flows, J. Wiley \& Sons, 1988.

[12] L. Bottura, A Numerical Model for the Simulation of Quench in the ITER Magnets, J. Comp. Phys., 125, 26-41,1996.

[13] L. Bottura, C. Rosso, Finite Element Simulation of Steady State and Forced Convection in Superfluid Helium, Int. J. Num. Methods Fluids, 30, 10911108, 1999.

[14] L. Bottura, Modelling the Compressible Flow in a CICC with Central Cooling Hole, NET Internal Report N/R/0821/38/B, 1993.

[15] C.A. Luongo, C.L. Chang, Helium Transport Phenomena in the $200 \mathrm{kA}$ SMES/CICC, Adv. Cryo. Eng., 39, 847-853, 1994.

[16] L. Bottura, M. Breschi, P.L. Ribani, Electromagnetic Analysis of Current Distribution in Multistrand Superconducting Cables, Inst. Phys. Conf. Ser., 167, 1191-1194, 2000.

[17] A. Akhmetov, L. Bottura, M. Breschi, P.L. Ribani, A Theoretical Investigation on Current Imbalance in Flat Two Layer Superconducting Cables, this issue. 
[18] O.C. Zienkiewicz, The Finite Element Method, 4th Edition, McGraw-Hill, 1991.

[19] R.M. Beam, R.F. Warming, Alternating Direction Implicit Methods for Parabolic Equations with a Mixed Derivative, SIAM J. Sci. Stat. Comp., 1, 131-159, 1980.

[20] L. Bottura, A. Shajii, Numerical Quenchback in Thermofluid Simulations of Superconducting Magnets, Int. J.Num. Methods Eng., 43, 1275-1293, 1998.

[21] Proceedings of CHATS-95, J. Fus. Energy, 14 (1), 1995.

[22] Proceedings of CHATS-97, C.A. Luongo ed., Cryogenics, 38 (5), 1998.

[23] L. Krempasky, C. Schmidt, Experimental Verification of "Supercurrents" in Superconducting Cables Exposed to AC-fields, Cryogenics, 39, 23-33, 1999.

[24] V.D. Arp, Stability and Thermal Quenches in Force-cooled Superconducting Cables, Proc. of 1980 Superconducting MHD Magnet Design Conference, MIT, 142-157, 1980. 


\section{Appendix A. Non-conservative flow equations}

The non-conservative form of the flow equations discussed in the text is convenient because the pressure appears explicitly. This improves the stability of the numerical solution, as stated in [12]. To obtain it we have followed Arp [24]. We start from the conservative form of the mass, momentum and energy conservation in a 1-D channel identified by the index $h$ :

$$
\begin{aligned}
& A_{h} \frac{\partial \rho_{h}}{\partial t}+\frac{\partial \rho_{h} V_{h}}{\partial x}=-\sum_{\substack{k=1 \\
k \neq h}}^{H} \Gamma_{h k}^{\rho} \\
& \frac{\partial \rho_{h} V_{h}}{\partial t}+\frac{\partial \rho_{h} V_{h} v_{h}}{\partial x}+A_{h} \frac{\partial p_{h}}{\partial x}=-A_{h} F_{h}-\sum_{\substack{k=1 \\
k \neq h}}^{H} \Gamma_{h k}^{v} \\
& \frac{\partial \rho_{h} A_{h} e_{h}}{\partial t}+\frac{\partial \rho_{h} V_{h} e_{h}}{\partial x}+\frac{\partial p_{h} V_{h}}{\partial x}=-\sum_{\substack{k=1 \\
k \neq h}}^{H} \Gamma_{h k}^{e}+\dot{q}_{h}^{\prime}+\dot{q}_{c f, h}^{\prime}
\end{aligned}
$$

where we have introduced the volumetric flux:

$V_{h}=A_{h} v_{h}$

To derive the non-conservative form we make use of the following thermodynamic relations between specific internal energy $i$, pressure $p$, density $\rho$ and temperature $T$ :

$$
\begin{aligned}
& d i=\left(\frac{p}{\rho}-\varphi C_{v} T\right) \frac{d \rho}{\rho}+C d T \\
& \left(c^{2}-\frac{p \varphi}{\rho}\right) d \rho=d p-\varphi \rho d i
\end{aligned}
$$

The relations involve the isentropic sound speed $c$, the specific heat at constant volume $C$ and the Gruneisen parameter $\varphi$. In addition we remember that the relation between total and internal specific energy is:

$e=i+\frac{v^{2}}{2}$

while the specific enthalpy $h$ is related to the internal specific energy by:

$h=i+\frac{p}{\rho}$ 
We start now with the momentum balance, Eq. (A.2), subtracting the continuity equation, Eq. (A.1), multiplied by the velocity, and we obtain the momentum balance in non-conservative form:

$$
\rho_{h} \frac{\partial V_{h}}{\partial t}+\frac{\rho_{h} V_{h}}{A_{h}} \frac{\partial V_{h}}{\partial x}+A_{h} \frac{\partial p_{h}}{\partial x}-\frac{\rho_{h} V_{h}^{2}}{A_{h}^{2}} \frac{\partial A_{h}}{\partial x}=-A_{h} F_{h}-\sum_{\substack{k=1 \\ k \neq h}}^{H}\left(\Gamma_{h k}^{v}-v_{h} \Gamma_{h k}^{\rho}\right)
$$

We now take the energy equation, and we explicitate the two terms forming the total energy, we subtract the continuity equation multiplied by $i_{h}+v_{h}^{2} / 2$, and the momentum balance multiplied by $v_{h}$ to obtain:

$$
A_{h} \rho_{h} \frac{\partial i_{h}}{\partial t}+\rho_{h} V_{h} \frac{\partial i_{h}}{\partial x}+p_{h} \frac{\partial V_{h}}{\partial x}=V_{h} F_{h}-\sum_{\substack{k=1 \\ k \neq h}}^{H}\left[\Gamma_{h k}^{e}-v_{h} \Gamma_{h k}^{v}-\left(i_{h}-\frac{v_{h}^{2}}{2}\right) \Gamma_{h k}^{\rho}\right]+\dot{q}_{h}^{\prime}+\dot{q}_{c f, h}^{\prime}(\mathrm{A} .10) .
$$

We can now use the relation (A.5) to substitute for the $d i_{h}$ differentials in Eq. (A.12), and we obtain:

$$
\begin{aligned}
& A_{h} \rho_{h} C_{h} \frac{\partial T_{h}}{\partial t}+\rho_{h} V_{h} C_{h} \frac{\partial T_{h}}{\partial x}+\frac{p_{h}}{\rho_{h}} \underline{\underline{\left(A_{h} \frac{\partial \rho_{h}}{\partial t}+\frac{\partial \rho_{h} V_{h}}{\partial x}\right)}}-\varphi_{h} C_{h} T_{h} \underline{\underline{\left(A_{h} \frac{\partial \rho_{h}}{\partial t}+V_{h} \frac{\partial \rho_{h}}{\partial x}\right)}}= \\
& =V_{h} F_{h}-\sum_{\substack{k=1 \\
k \neq h}}^{H}\left[\Gamma_{h k}^{e}-v_{h} \Gamma_{h k}^{v}-\left(i_{h}-\frac{v_{h}^{2}}{2}\right) \Gamma_{h k}^{\rho}\right]+\dot{q}_{h}^{\prime}+\dot{q}_{c f, h}^{\prime}
\end{aligned}
$$

that can be reduced using the continuity equation (A.1) to substitute the terms underlined and to obtain the desired energy balance in non-conservative form:

$$
\begin{aligned}
& A_{h} \rho_{h} C_{h} \frac{\partial T_{h}}{\partial t}+\rho_{h} V_{h} C_{h} \frac{\partial T_{h}}{\partial x}+\rho_{h} \varphi_{h} C_{h} T_{h} \frac{\partial V_{h}}{\partial x}= \\
& V_{h} F_{h}-\sum_{\substack{k=1 \\
k \neq h}}^{H}\left[\Gamma_{h k}^{e}-v_{h} \Gamma_{h k}^{v}-\left(h_{h}-\frac{v_{h}^{2}}{2}-\varphi_{h} C_{h} T_{h}\right) \Gamma_{h k}^{\rho}\right]+\ddot{q}_{h}^{\prime}+\ddot{q}_{c f, h}^{\prime}
\end{aligned}
$$

A third equation is needed, the non-conservative form of the continuity balance. This is obtained substituting the $d \rho_{h}$ differential using the thermodynamic identity (A.6) in the continuity equation (A.1):

$$
\begin{aligned}
& A_{h} \frac{\partial p_{h}}{\partial t}+V_{h} \frac{\partial p_{h}}{\partial x}+\rho_{h} c_{h}^{2} \frac{\partial V_{h}}{\partial x}+\rho_{h} c_{h}^{2} v_{h} \frac{\partial A_{h}}{\partial x}-\varphi_{h} \underline{\underline{\left(A_{h} \rho_{h} \frac{\partial i_{h}}{\partial t}-\rho_{h} V_{h} \frac{\partial i_{h}}{\partial x}-p_{h} \frac{\partial V_{h}}{\partial x}\right)}} \\
& -\sum_{\substack{k=1 \\
k \neq h}}^{H}\left(c_{h}^{2}-\frac{p_{h} \varphi_{h}}{\rho_{h}}\right) \Gamma_{h k}^{\rho}
\end{aligned}
$$


We reduce further the equation above, in particular the terms underlined, adding the non-conservative intermediate form of the energy equation Eq. (A.10) multiplied by $\varphi_{h^{\prime}}$ and we obtain:

$$
\begin{aligned}
& A_{h} \frac{\partial p_{h}}{\partial t}+V_{h} \frac{\partial p_{h}}{\partial x}+\rho_{h} c_{h}^{2} \frac{\partial V_{h}}{\partial x}= \\
& -\sum_{\substack{k=1 \\
k \neq h}}^{H}\left\{c_{h}^{2} \Gamma_{h k}^{\rho}+\varphi_{h}\left[\Gamma_{h k}^{e}-v_{h} \Gamma_{h k}^{v}-\left(h_{h}-\frac{v_{h}^{2}}{2}\right) \Gamma_{h k}^{\rho}\right]\right\}+\varphi_{h} V_{h} F_{h}+\varphi_{h} \dot{q}_{h}^{\prime}+\varphi_{h} \dot{q}_{c f, h}^{\prime}
\end{aligned}
$$

that is the desired non-conservative continuity balance. 


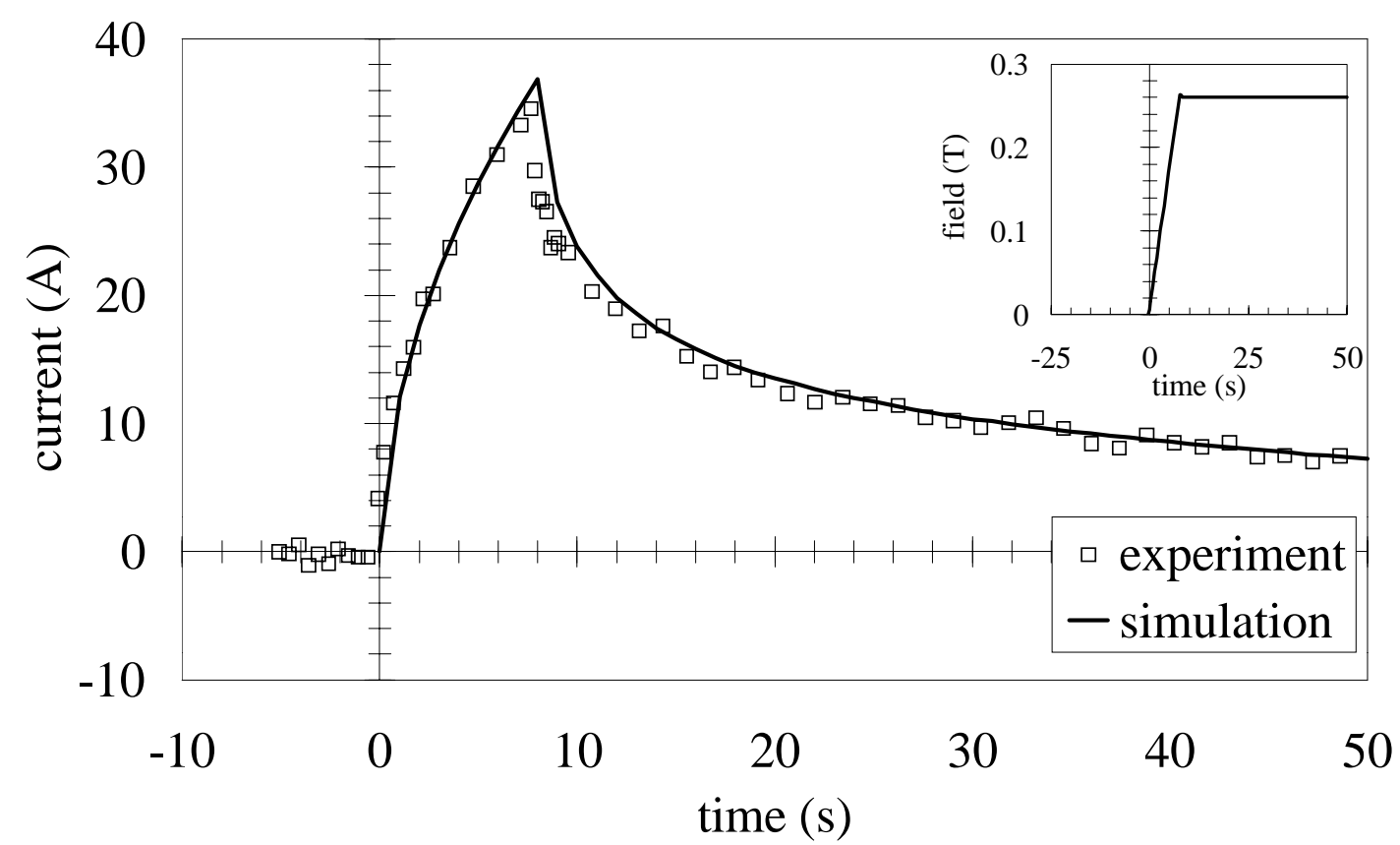

Figure 1. Measured and simulated supercurrent generated by a field ramp in the experiment of Krempasky and Schmidt [23]. Sample length $4.7 \mathrm{~m}$, field ramp as shown in the inset.

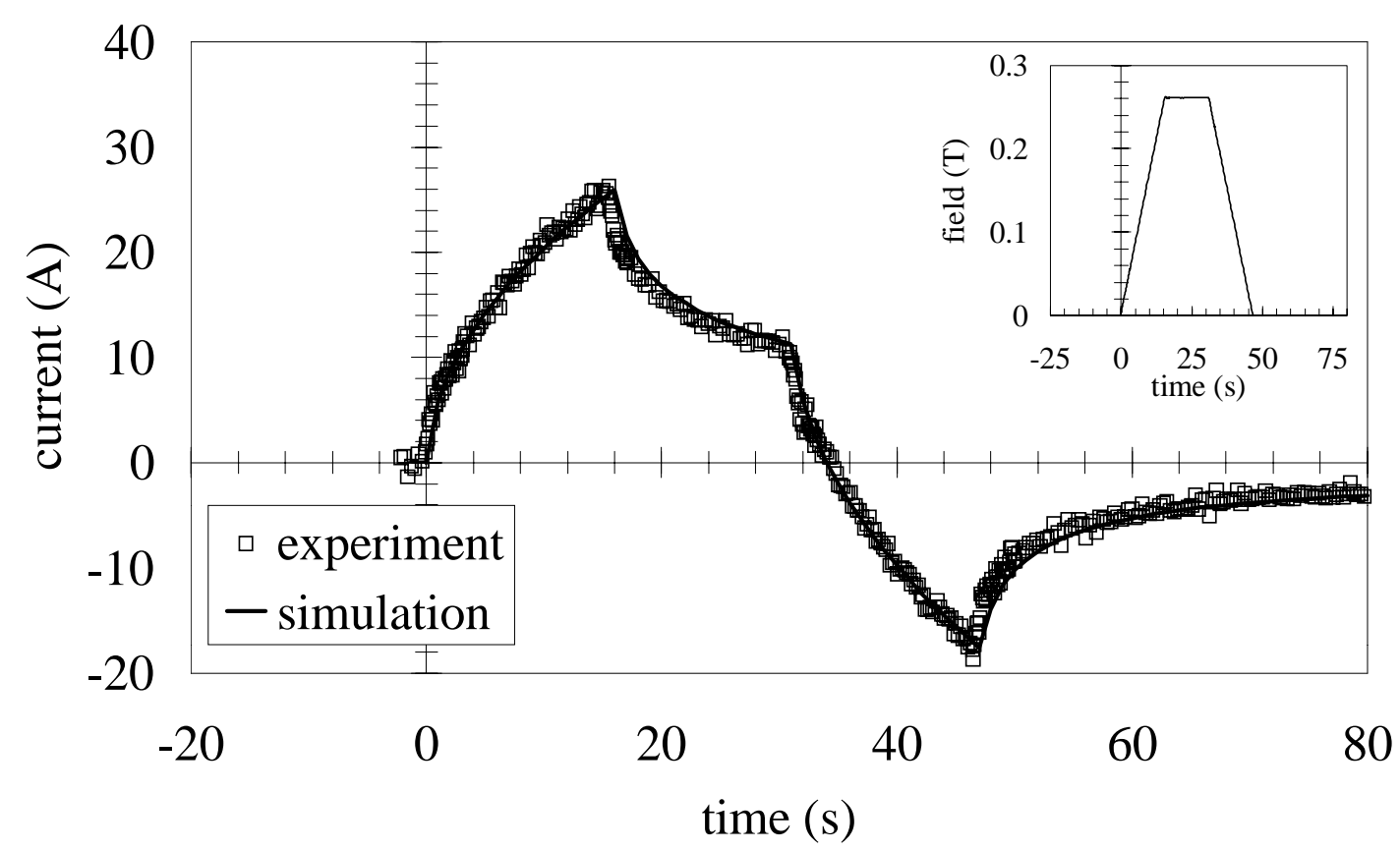

Figure 2. Measured and simulated supercurrent generated by a field sweep in the experiment of Krempasky and Schmidt [23]. Conditions as in Fig. 1, but trapezoidal field sweep as shown in the inset. 


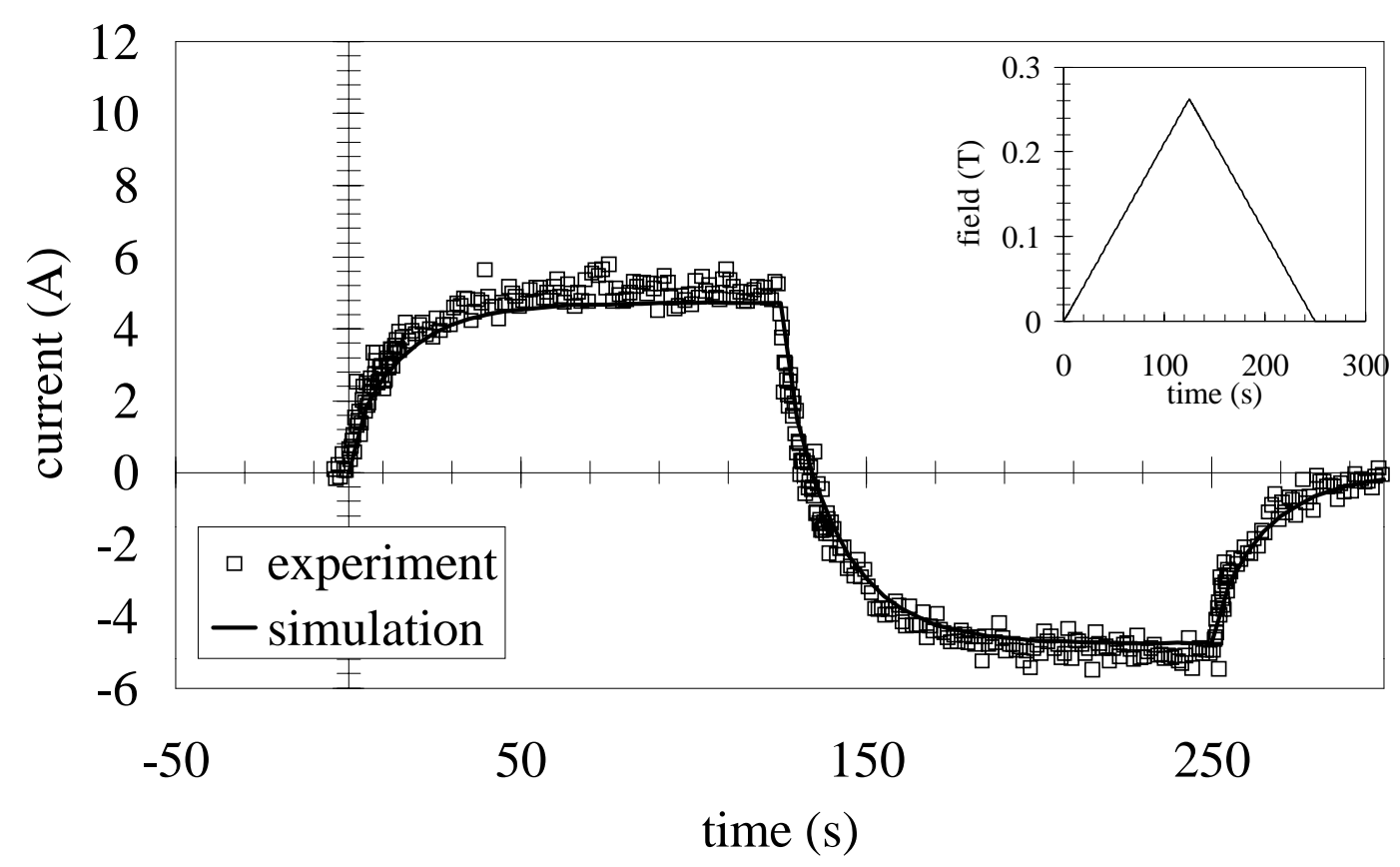

Figure 3. Measured and simulated steady state supercurrent generated by a slow field sweep in the experiment of Krempasky and Schmidt [23]. Sample length $2.35 \mathrm{~m}$, trapezoidal field sweep as shown in the inset.

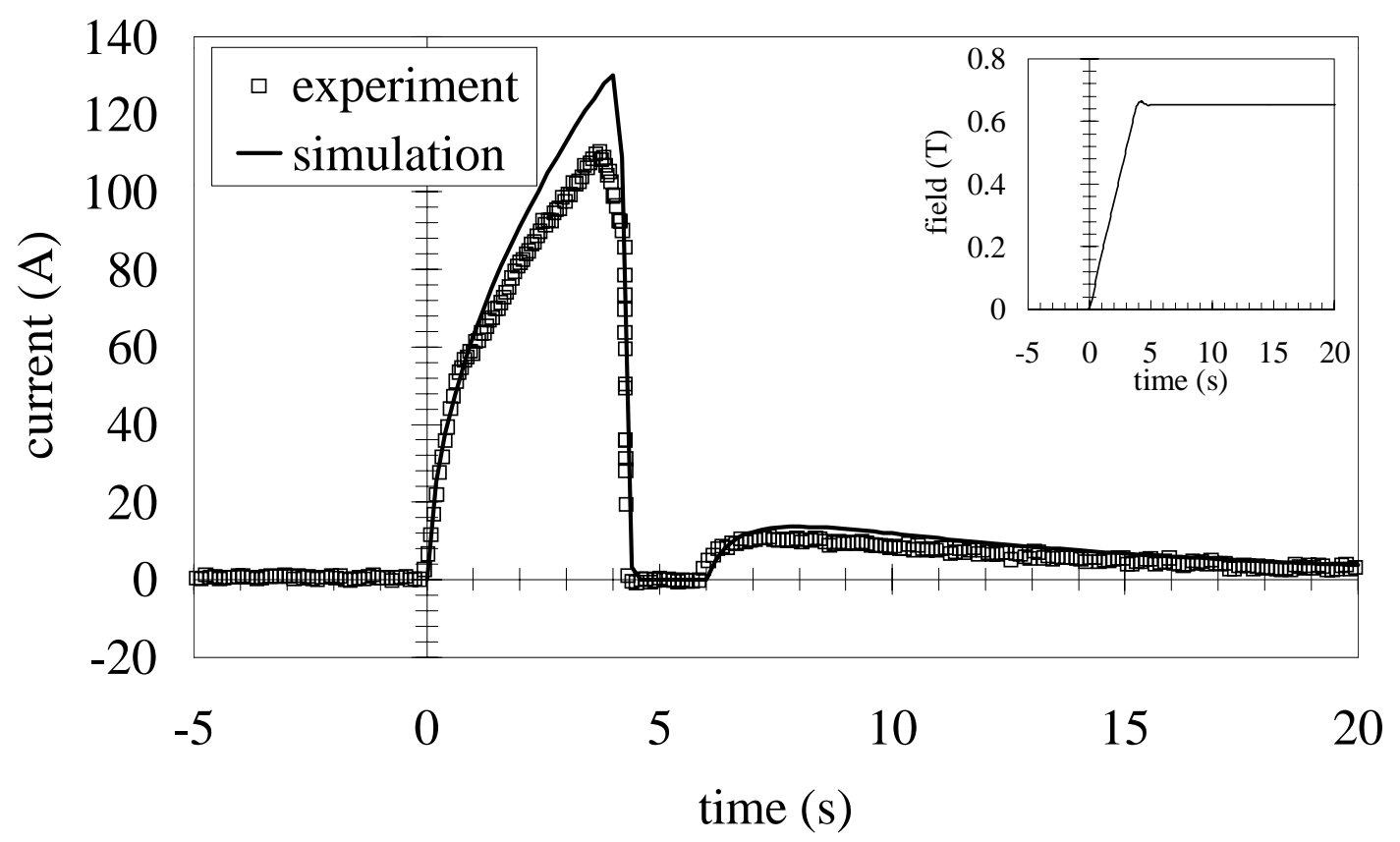

Figure 4. Measured and simulated supercurrent generated by a field ramp in the experiment of Krempasky and Schmidt [23]. Sample length 2.35 $\mathrm{m}$, fast field ramp as shown in the inset. The center heater was fired at approximately $4.25 \mathrm{~s}$, for a total of $1.6 \mathrm{~s}$. 